\title{
Some optimal control problems of heat equations with weighted controls
}

\section{Shufang Liu*, Dandan Liu and Guoqi Wang}

${ }^{*}$ Correspondence:

math_Isf@csu.edu.cn

School of Mathematics and

Statistics, Central South University,

Changsha, 410075, P.R. China

\begin{abstract}
In this paper, the time and norm optimal control problems of controlled heat equations with a weight function are considered. For the time optimal problems, we study the following two cases: one is for equations with multi-domain control under null controllability, and the other is for equations under approximate null controllability. We prove the solvability, and obtain the bang-bang principle of the time optimal controls for aforementioned both cases. For the norm optimal control problems, we focus on equations with multi-time and multi-domain control, and present the solvability of these problems.
\end{abstract}

MSC: $35 \mathrm{~K} 05 ; 49 J 20$

Keywords: heat equation; weight function; optimal control problem; multi-domain

\section{Introduction}

Let $T$ be a positive number and $\Omega$ be an open bounded domain with smooth boundary in $\mathbb{R}^{N}, N \geq 1$. Let $K \in \mathbb{Z}^{+},\left\{E_{i}\right\} \equiv\left\{E_{i}\right\}_{i=1}^{K}$ be a sequence of Lebesgue measurable subsets of $(0, T)$ and $\left\{\omega_{i}\right\} \equiv\left\{\omega_{i}\right\}_{i=1}^{K}$ be a sequence of positive Lebesgue measurable subsets of $\Omega$ with $\omega_{i} \cap \omega_{j}=\emptyset$, for all $i, j \in\{1,2, \ldots, K\}$ and $i \neq j$. Denote by $\chi_{E_{i}}, \chi_{\omega_{i}}$ the characteristic function of $E_{i}, \omega_{i}$, respectively, for each $i \in\{1,2, \ldots, K\}$. Consider the following controlled heat equation with a weight function:

$$
\begin{cases}\partial_{t} y(x, t)-\Delta y(x, t)=\rho(x) \sum_{i=1}^{K} \chi_{E_{i}}(t) \chi_{\omega_{i}}(x) u_{i}(x, t), & \text { in } \Omega \times(0, T), \\ y(x, t)=0, & \text { on } \partial \Omega \times(0, T), \\ y(x, 0)=y_{0}(x), & \text { in } \Omega,\end{cases}
$$

where $\rho \in L^{2}(\Omega)$ is a weight function satisfying $0<\rho(x) \leq 1$ for a.e. $x \in \Omega$, and $0 \neq$ $y_{0} \in L^{2}(\Omega)$ is a given function. We denote the solution to (1.1) by $y\left(\cdot, ;\left\{\chi_{E_{i}} \chi_{\omega_{i}} u_{i}\right\}, y_{0}\right)$. For simplicity, when $E_{i}=(0, T)$ for all $i \in\{1,2, \ldots, K\}$, we write $y\left(\cdot, \cdot ;\left\{\chi_{\omega_{i}} u_{i}\right\}, y_{0}\right)$ for $y\left(\cdot, \cdot ;\left\{\chi_{E_{i}} \chi_{\omega_{i}} u_{i}\right\}, y_{0}\right)$; furthermore, when $K=1$, write $\omega, y\left(\cdot, \cdot ; \chi_{\omega} u, y_{0}\right)$ for $\omega_{i}, y\left(\cdot, \cdot ;\left\{\chi_{\omega_{i}} u_{i}\right\}\right.$, $\left.y_{0}\right)$, respectively.

The weight function $\rho$ in equation (1.1) is meaningful, which stands for the different influence of the control function in different location.

As is well known, optimization is one of the most important problems in control theory and there exist some work on this topic (see, e.g., [1-3]). Roughly speaking, the goal of op-

(c) The Author(s) 2017. This article is distributed under the terms of the Creative Commons Attribution 4.0 International License (http://creativecommons.org/licenses/by/4.0/), which permits unrestricted use, distribution, and reproduction in any medium, provided you give appropriate credit to the original author(s) and the source, provide a link to the Creative Commons license, and indicate if changes were made. 
timization is to improve a variable in order to maximize a benefit (or minimize a cost). The time and norm optimal control problems are important and interesting branches of optimization. For the deterministic systems, the reader can refer [4] to obtain recent results and find open problems. The reader can also refer [5-10] for controlled heat equations. For the stochastic ones, the norm optimal control problems were considered in $[11,12]$ for controlled stochastic ordinary differential equations, and in [13] for controlled stochastic heat equations.

In this paper, we shall consider the time and norm optimal control problems of heat equations with a weight function. In Section 2, we consider two kind time optimal control problems: one is for equations with multi-domain control under null controllability, and the other is for equations under approximate null controllability. We obtain the bang-bang principle of the time optimal controls for these two problems. In Section 3, we consider the norm optimal problems with multi-time and multi-domain control, and we obtain the solvability of these problems.

\section{Time optimal control problems}

In this section, we first state two time optimal control problems, and then study the solvability of these problems, obtain the bang-bang property of the time optimal controls. Throughout this section, for all $i \in\{1,2, \ldots, K\}, E_{i}=(0, T)$, and

$$
u_{i} \in \mathcal{U}_{\mathrm{ad}}^{i} \equiv\left\{u \in L^{\infty}\left(0,+\infty ; L^{2}(\Omega)\right) \mid\|u(t)\|_{L^{2}(\Omega)} \leq M_{i} \text { a.e. } t \in(0,+\infty)\right\} .
$$

When $K=1$, for simplicity, we write $\mathcal{U}_{\text {ad }}$ for $\mathcal{U}_{\text {ad }}^{i}$.

In the following, we consider the following two time optimal control problems subject to (1.1):

\section{Problem (TP1)}

$$
T^{*}=\inf \left\{T \mid y\left(\cdot, T ;\left\{\chi_{\omega_{i}} u_{i}\right\}, y_{0}\right)=0, u_{i} \in \mathcal{U}_{\text {ad }}^{i} \text { for all } i \in\{1,2, \ldots, K\}\right\}
$$

Problem (TP2) For $K=1$,

$$
T_{\varepsilon}^{*}=\inf \left\{T \mid y\left(\cdot, T ; \chi_{\omega} u, y_{0}\right) \in \bar{B}(0, \varepsilon), u \in \mathcal{U}_{\mathrm{ad}}\right\}
$$

Here and in what follows, we denote by $B(u, r)$ the open ball in $L^{2}(\Omega)$ with center $u \in L^{2}(\Omega)$ and radius $r>0$, and by $\bar{B}(u, r)$ the closed ball in $L^{2}(\Omega)$ with center $u \in L^{2}(\Omega)$ and radius $r>0$.

In order to obtain the solvability of Problem (TP1), we assume that there exists a constant $M>0$ such that

$$
M_{i} \leq M \quad \text { for all } i \in\{1,2, \ldots, K\}
$$

Notice that the hypothesis (2.2) is reasonable: for a single control system

$$
\begin{cases}\partial_{t} y(x, t)-\Delta y(x, t)=\rho(x) \chi_{\omega}(x) u(x, t), & \text { in } \Omega \times(0,+\infty), \\ y(x, t)=0, & \text { on } \partial \Omega \times(0,+\infty), \\ y(x, 0)=y_{0}(x), & \text { in } \Omega,\end{cases}
$$


its optimal time

$$
T^{*} \equiv \inf \left\{T \mid y\left(\cdot, T ; \chi_{\omega} u, y_{0}\right)=0, u \in \mathcal{U}_{\mathrm{ad}}\right\} \rightarrow 0
$$

as $M \rightarrow \infty$.

It is obvious that Problem (TP1) is related to null controllable problem of (1.1), while Problem (TP2) is related to approximately controllable problem of (1.1). It is well known that, when $K=1$ and $\rho \equiv 1$, the system (1.1) is null controllable for the measurable control domain $\omega$ (see [5]), even if the characteristic function $\chi_{\omega}$ can be relaxed by a measurable function $\beta \in L^{2}(\Omega)$ with $0 \leq \beta \leq 1$ for a.e. $x \in \Omega$ and $\int_{\Omega} \beta^{2}(x) d x=\alpha|\Omega|$ (see [6]). Here $\alpha \in(0,1)$ is a given constant and $|\Omega|$ is the Lebesgue measure of $\Omega$. It is natural that there exist a positive constant $T$ and a control $u \in L^{\infty}\left(0, T ; L^{2}(\Omega)\right)$ such that $y\left(x, T ; u, y_{0}\right)=0$ (see $[5,6])$.

The following result is related to the solvability of Problem (TP1).

Theorem 2.1 Let $\left\{M_{i}\right\}$ be a given positive real number sequence satisfying (2.2). Then there exists $T^{*}>0$, such that $T^{*}$ is the solution to Problem (TP1). Moreover, for each $i=1,2, \ldots, K$, there exists a unique $u_{i}^{*} \in L^{\infty}\left(0, T^{*} ; L^{2}(\Omega)\right)$, such that

$$
\left\|u_{i}^{*}\right\|_{L^{2}(\Omega)}=M_{i} \quad \text { for a.e. } t \in\left(0, T^{*}\right) \text { with } M_{i} \leq M \text { for all } i \in\{1,2, \ldots, K\},
$$

i.e., the time optimal controls sequence of Problem (TP1) has the bang-bang property.

The following lemma is needed in proving Theorem 2.1, which comes from [5, 9].

Lemma 2.2 Let $E \subset[0, T]$ and $\omega \subset \Omega$ be two positive measurable sets. Then, for each $y_{0} \in L^{2}(\Omega)$, there is a bounded control function $u(\cdot) \in L^{\infty}\left(0, T ; L^{2}(\Omega)\right)$ with

$$
\|u\|_{L^{\infty}\left(0, T ; L^{2}(\Omega)\right)} \leq C\left\|y_{0}\right\|_{L^{2}(\Omega)},
$$

such that the solution to the equation

$$
\begin{cases}\partial_{t} y(x, t)-\Delta y(x, t)=\rho(x) \chi_{E}(t) \chi_{\omega}(x) u(x, t), & \text { in } \Omega \times(0, T), \\ y(x, t)=0, & \text { on } \Omega \times(0, T) \\ y(x, 0)=y_{0}(x), & \text { in } \Omega,\end{cases}
$$

satisfies $y\left(\cdot, T ; u, y_{0}\right)=0$. Here $C=C(\Omega, T,|E|,|\omega|)$ is a constant.

We are now in the position to prove Theorem 2.1.

Proof of Theorem 2.1 Since the proof is long, we separate it into two steps.

Step 1. For fixed $i_{0} \in\{1,2, \ldots, K\}$, consider the following system:

$$
\begin{cases}\partial_{t} y(x, t)-\Delta y(x, t)=\rho(x) \chi_{\omega_{i_{0}}}(x) u_{i_{0}}(x, t), & \text { in } \Omega \times(0,+\infty), \\ y(x, t)=0, & \text { on } \partial \Omega \times(0,+\infty), \\ y(x, 0)=y_{0}(x), & \text { in } \Omega .\end{cases}
$$


By Lemma 2.1 of [6], we know that there exist a control $u_{i_{0}} \in \mathcal{U}_{\mathrm{ad}}^{i_{0}}$ and $T$, such that $y\left(\cdot, T, \chi_{\omega_{i}} u_{i_{0}}, y_{0}\right)=0$. we also know that $0 \neq y_{0} \in L^{2}(\Omega)$ is a given function. Therefore,

$$
\begin{aligned}
0 & <T^{*} \equiv \inf \left\{T \mid y\left(\cdot, T ;\left\{\chi_{\omega_{i}} u_{i}\right\}, y_{0}\right)=0, u^{i} \in \mathcal{U}_{\text {ad }}^{i} \text { for all } i \in\{1,2, \ldots, K\}\right\} \\
& \leq \inf \left\{T \mid y\left(\cdot, T ; \chi_{\omega_{i_{0}}} u_{i_{0}}, y_{0}\right)=0, u_{i_{0}} \in \mathcal{U}_{\text {ad }}^{i_{0}}\right\}<\infty
\end{aligned}
$$

Hence, there exists a sequence $\left\{T_{n}\right\}$, such that $\left\{T_{n}\right\}$ is a monotone decreasing sequence with $y\left(\cdot, T_{n} ;\left\{\chi_{\omega_{i}} u_{i}^{n}\right\}, y_{0}\right)=0$ and

$$
T_{n} \rightarrow T^{*} \equiv \inf \left\{T \mid y\left(\cdot, T ;\left\{\chi_{\omega_{i}} u_{i}\right\}, y_{0}\right)=0, u_{i} \in \mathcal{U}_{\mathrm{ad}}^{i} \text { for all } i \in\{1,2, \ldots, K\}\right\}
$$

Without loss of generality, we assume that $T_{n} \leq T^{*}+1$ for all $n \in \mathbb{N}$. Then $y^{n} \equiv$ $y\left(\cdot, \cdot ;\left\{\chi_{\omega_{i}} u_{i}^{n}\right\}, y_{0}\right)$ is a solution to the following equation:

$$
\begin{cases}\partial_{t} y^{n}(x, t)-\Delta y^{n}(x, t)=\rho(x) \sum_{i=1}^{K} \chi_{\omega_{i}}(x) u_{i}^{n}(x, t), & \text { in } \Omega \times\left(0, T_{n}\right), \\ y^{n}(x, t)=0, & \text { on } \partial \Omega \times\left(0, T_{n}\right), \\ y^{n}(x, 0)=y_{0}(x), y^{n}\left(x, T_{n}\right)=0, & \text { in } \Omega .\end{cases}
$$

Now, denote

$$
\tilde{u}_{i}^{n}(x, t)= \begin{cases}u_{i}^{n}(x, t), & (x, t) \in \Omega \times\left(0, T_{n}\right) \\ 0, & (x, t) \in \Omega \times\left[T_{n},+\infty\right)\end{cases}
$$

Then

$$
\tilde{y}^{n}(x, t)= \begin{cases}y^{n}(x, t), & (x, t) \in \Omega \times\left(0, T_{n}\right), \\ 0, & (x, t) \in \Omega \times\left[T_{n},+\infty\right)\end{cases}
$$

solves the following system:

$$
\begin{cases}\partial_{t} \tilde{y}^{n}(x, t)-\Delta \tilde{y}^{n}(x, t)=\rho(x) \sum_{i=1}^{K} \chi_{\omega_{i}}(x) \tilde{u}_{i}^{n}(x, t), & \text { in } \Omega \times(0,+\infty), \\ \tilde{y}^{n}(x, t)=0, & \text { on } \partial \Omega \times(0,+\infty), \\ \tilde{y}^{n}(x, 0)=y_{0}(x), & \text { in } \Omega, \\ \tilde{y}_{n}(x, t)=0, & \text { in } \Omega \times\left[T_{n},+\infty\right) .\end{cases}
$$

Moreover, by the definition of $\tilde{y}^{n}$, it is easy to see that $\tilde{y}^{n}$ solves the following system:

$$
\begin{cases}\partial_{t} \tilde{y}^{n}(x, t)-\Delta \tilde{y}^{n}(x, t)=\rho(x) \sum_{i=1}^{K} \chi_{\omega_{i}}(x) \tilde{u}_{i}^{n}(x, t), & \text { in } \Omega \times\left(0, T^{*}+1\right), \\ \tilde{y}^{n}(x, t)=0, & \text { on } \partial \Omega \times\left(0, T^{*}+1\right), \\ \tilde{y}^{n}(x, 0)=y_{0}(x), & \text { in } \Omega, \\ \tilde{y}_{n}(x, t)=0, & \text { in } \Omega \times\left[T_{n}, T^{*}+1\right) .\end{cases}
$$


Note that $\left\|\tilde{u}_{1}^{n}\right\|_{L^{2}(\Omega)} \leq M_{1}$ for all $n \in \mathbb{N}$. Then there exist a subsequence $\left\{\tilde{u}_{1}^{n_{1}}\right\} \subset L^{\infty}\left(0, T^{*}+\right.$ $\left.1 ; L^{2}(\Omega)\right)$ of $\left\{\tilde{u}_{1}^{n}\right\}$ and $\tilde{u}_{1}^{0} \in L^{\infty}\left(0, T^{*}+1 ; L^{2}(\Omega)\right)$ such that

$$
\tilde{u}_{1}^{n_{1}} \rightarrow \tilde{u}_{1}^{0} \quad \text { weakly* in } L^{\infty}\left(0, T^{*}+1 ; L^{2}(\Omega)\right) \text { as } n_{1} \rightarrow \infty .
$$

Similarly, since $\left\|u_{2}^{n_{1}}\right\|_{L^{2}(\Omega)} \leq M_{2}$ for all $n_{1} \in \mathbb{N}$, there exist a subsequence $\left\{\tilde{u}_{2}^{n_{2}}\right\}$ of $\left\{\tilde{u}_{2}^{n_{1}}\right\}$ and $\tilde{u}_{2}^{0} \in L^{\infty}\left(0, T^{*}+1 ; L^{2}(\Omega)\right)$ such that

$$
\tilde{u}_{2}^{n_{2}} \rightarrow \tilde{u}_{2}^{0} \quad \text { weakly* in } L^{\infty}\left(0, T^{*}+1 ; L^{2}(\Omega)\right) \text { as } n_{2} \rightarrow \infty .
$$

By inductive argument, for each $i \in\{1,2, \ldots, K\}$, there exist a subsequence $\left\{\tilde{u}_{i}^{n_{i}}\right\}$ of $\left\{\tilde{u}_{i}^{n_{i-1}}\right\}$ and $\tilde{u}_{i}^{0} \in L^{\infty}\left(0, T^{*}+1 ; L^{2}(\Omega)\right)$ such that

$$
\tilde{u}_{i}^{n_{i}} \rightarrow \tilde{u}_{i}^{0} \quad \text { weakly* in } L^{\infty}\left(0, T^{*}+1 ; L^{2}(\Omega)\right) \text { as } n_{i} \rightarrow \infty
$$

By the diagram argument, for all $i \in\{1,2, \ldots, K\}$, we can abstract a subsequence $\left\{\tilde{u}_{i}^{n_{n}}\right\}$ of $\left\{\tilde{u}_{i}^{n}\right\}$ such that

$$
\tilde{u}_{i}^{n_{n}} \rightarrow \tilde{u}_{i}^{0} \quad \text { weakly }{ }^{*} \text { in } L^{\infty}\left(0, T^{*}+1 ; L^{2}(\Omega)\right) \text { as } n \rightarrow \infty .
$$

Since $\omega_{i} \cap \omega_{j}=\emptyset$ for all $i, j \in\{1,2, \ldots, K\}$ with $i \neq j$, one can get

$$
\rho(x) \sum_{i=1}^{K} \chi_{\omega_{i}} \tilde{u}_{i}^{n_{n}} \rightarrow \rho(x) \sum_{i=1}^{K} \chi_{\omega_{i}} \tilde{u}_{i}^{0} \quad \text { weakly* in } L^{\infty}\left(0, T^{*}+1 ; L^{2}(\Omega)\right) \text { as } n \rightarrow \infty .
$$

On the other hand, $\tilde{y}^{n_{n}}$ is the solution to the following system:

$$
\begin{cases}\partial_{t} \tilde{y}^{n_{n}}(x, t)-\Delta \tilde{y}^{n_{n}}(x, t)=\rho(x) \sum_{i=1}^{K} \chi_{\omega_{i}}(x) \tilde{u}_{i}^{n_{n}}(x, t), & \text { in } \Omega \times\left(0, T^{*}+1\right), \\ \tilde{y}^{n_{n}}(x, t)=0, & \text { on } \partial \Omega \times\left(0, T^{*}+1\right), \\ \tilde{y}^{n_{n}}(x, 0)=y_{0}(x), & \text { in } \Omega, \\ \tilde{y}_{n_{n}}(x, t)=0, & \text { in } \Omega \times\left[T_{n_{n}}, T^{*}+1\right) .\end{cases}
$$

Then there exist a subsequence of $\left\{\tilde{y}^{n_{n}}\right\}$, still so denoted, and $\tilde{y}^{0}$ such that

$$
\tilde{y}^{n_{n}} \rightarrow \tilde{y}^{0} \quad \text { weakly in } L^{2}\left(\Omega \times\left(0, T^{*}+1\right)\right) \text { as } n \rightarrow \infty
$$

and

$$
\begin{cases}\partial_{t} \tilde{y}^{0}(x, t)-\Delta \tilde{y}^{0}(x, t)=\rho(x) \sum_{i=1}^{K} \chi_{\omega_{i}}(x) \tilde{u}_{i}^{0}(x, t), & \text { in } \Omega \times\left(0, T^{*}+1\right), \\ \tilde{y}^{0}(x, t)=0, & \text { on } \partial \Omega \times\left(0, T^{*}+1\right), \\ \tilde{y}^{0}(x, 0)=y_{0}(x), & \text { in } \Omega .\end{cases}
$$

Note that $\tilde{y}^{n_{n}}=0$ in $\Omega \times\left[T_{n_{n}}, T^{*}+1\right)$. Since for $n \in \mathbb{N}$, one has $\tilde{y}^{n_{n}}=0$ in $\Omega \times\left[T_{n_{n}}, T^{*}+1\right)$,

$$
\tilde{y}^{n_{n}} \rightarrow \tilde{y}^{0} \quad \text { in } L^{2}\left(\Omega \times\left(0, T^{*}+1\right)\right) \text { as } n \rightarrow \infty .
$$


We get $\tilde{y}^{0}=0$ in $\Omega \times\left[T_{n_{n}}, T^{*}+1\right)$. We get $\tilde{y}^{0}=0$ in $\left[T^{*}, T^{*}+1\right)$ since $T_{n_{n}} \rightarrow T^{*}$ as $n \rightarrow \infty$. Take

$$
u_{i}^{0}=\left.\tilde{u}_{i}^{0}\right|_{\Omega \times\left(0, T^{*}\right)}, \quad \text { and } \quad y^{0}=\left.\tilde{y}^{0}\right|_{\Omega \times\left(0, T^{*}\right)} .
$$

By the fact that $\tilde{y}^{0} \in C\left((0, T] ; L^{2}(\Omega)\right), y^{0}$ is the solution to the following system:

$$
\begin{cases}\partial_{t} y^{0}(x, t)-\Delta y^{0}(x, t)=\rho(x) \sum_{i=1}^{K} \chi_{\omega_{i}}(x) u_{i}^{0}(x, t), & \text { in } \Omega \times\left(0, T^{*}\right), \\ y^{0}(x, t)=0, & \text { on } \partial \Omega \times\left(0, T^{*}\right), \\ y^{0}(x, 0)=y_{0}(x), & y^{0}\left(x, T^{*}\right)=0,\end{cases}
$$

which implies that $\left\{u_{i}^{0}\right\}$ are the desired controls respect to the optimal time $T^{*}$.

Step 2. In the following, we shall show that the time optimal control of Problem (TP1) has the bang-bang property. Otherwise, we suppose that there exist $i_{0} \in\{1,2, \ldots, K\}$ and a subset $E^{0} \subset\left[\alpha, T^{*}-\alpha\right]$ with positive measure for some $\alpha>0$ and a positive number $\varepsilon_{0}$, such that

$$
u_{i_{0}}^{*} \in \mathcal{U}_{\mathrm{ad}}^{i_{0}} \quad \text { and } \quad M_{i_{0}}-\left\|u_{i_{0}}^{*}\right\|_{L^{2}(\Omega)} \geq \varepsilon_{0}, \quad \text { for each } t \text { in the set } E^{0},
$$

where $u_{i_{0}}^{*}$ is the time optimal control respect to $T^{*}$. It is obvious that the solution to (1.1) satisfies

$$
y\left(\cdot, T^{*} ;\left\{\chi_{\omega_{i}} u_{i}^{*}\right\}, y_{0}\right)=0
$$

and, for each $t \in E^{0}, B\left(u_{i_{0}}^{*}(t), \frac{\varepsilon_{0}}{2}\right) \subset B\left(0, M_{i_{0}}\right)$.

We denote by $e^{\Delta t}$ the semigroup generated by $\Delta$ with the Dirichlet boundary condition. Set

$$
h_{\delta}=\int_{0}^{\delta} e^{\Delta(\delta-\sigma)} \rho \sum_{i=1}^{K} \chi_{\omega_{i}} u_{i}^{*}(\sigma) d \sigma+\left(e^{\Delta \delta}-I\right) y_{0} .
$$

Considering the following system:

$$
\begin{cases}z_{t}^{\delta}(x, t)-\Delta z^{\delta}(x, t)=\rho(x) \chi_{E_{\delta}^{0}}(t) \chi_{\omega_{i_{0}}}(x) w_{\delta}(x, t), & \text { in } \Omega \times\left(0, T^{*}-\delta\right), \\ z(x, t)=0, & \text { on } \partial \Omega\left(0, T^{*}-\delta\right), \\ z(x, 0)=-h_{\delta}(x), & \text { in } \Omega,\end{cases}
$$

where $E_{\delta}^{0}$ is the set $\left\{t \mid t+\delta \in E^{0}\right\}$. By Lemma 2.2, there exist positive constants $\delta_{1}$ and $L=L\left(\Omega, T,\left|E_{0}\right|,\left|\omega_{i_{0}}\right|\right)$, such that, for each $\delta$ with $0<\delta \leq \delta_{1}$, there is a control $w_{\delta}$ in the space $L^{\infty}\left(0, T^{*}-\delta ; L^{2}(\Omega)\right)$ with the estimate

$$
\left\|w_{\delta}\right\|_{L^{\infty}\left(0, T^{*}-\delta ; L^{2}(\Omega)\right)} \leq L\left\|h_{\delta}\right\|_{L^{2}(\Omega)}
$$

and the solution to (2.5) satisfies

$$
z^{\delta}\left(\cdot, T^{*}-\delta ; \omega_{\delta},-h_{\delta}\right)=0
$$


On the other hand, by (2.4), there exists a positive number $\delta_{2}$ such that, for each positive number $\delta$ with $\delta \leq \delta_{2}$, one has

$$
\left\|h_{\delta}\right\|_{L^{2}(\Omega)} \leq \frac{\varepsilon_{0}}{2 L} .
$$

Therefore, for each $\delta \leq \delta_{0} \equiv \min \left\{\delta_{1}, \delta_{2}\right\}$, there exists a control $w_{\delta}$ satisfying

$$
\left\|w_{\delta}\right\|_{L^{\infty}\left(0, T^{*}-\delta ; L^{2}(\Omega)\right)} \leq \frac{\varepsilon_{0}}{2}
$$

and the corresponding solution to (2.5) satisfies (2.6).

Set

$$
v_{i}(x, t)= \begin{cases}u_{i}^{*}(x, \delta+t)+w_{\delta}(x, t), & \text { for } i=i_{0} \text { and }(x, t) \in \Omega \times E_{\delta}^{0} \\ u_{i}^{*}(x, \delta+t), & \text { for } i=i_{0} \text { and }(x, t) \in \Omega \times\left(\left(0, T^{*}-\delta\right)-E_{\delta}^{0}\right), \\ u_{i}^{*}(x, \delta+t), & \text { for } i \neq i_{0} \text { and }(x, t) \in \Omega \times\left(0, T^{*}-\delta\right), \\ 0, & \text { otherwise. }\end{cases}
$$

It is obvious that $v_{i} \in \mathcal{U}_{\text {ad }}^{i}$ for all $i \in\{1,2, \ldots, K\}$. Consider the following system:

$$
\begin{cases}y_{t}(x, t)-\Delta y(x, t)=\rho(x) \sum_{i=1}^{K} \chi_{\omega_{i}}(x) v_{i}(x, t), & \text { in } \Omega \times(0,+\infty), \\ y(x, t)=0, & \text { on } \partial \Omega \times(0,+\infty), \\ y(x, 0)=y_{0}(x), & \text { in } \Omega .\end{cases}
$$

For any $0<\delta<\min \left\{\frac{T^{*}}{2}, \delta_{0}\right\}$, it is easy to check that the solution to above equation satisfies

$$
\begin{aligned}
y\left(\cdot, T^{*}-\delta ;\left\{v_{i}\right\}, y_{0}\right)= & e^{\Delta\left(T^{*}-\delta\right)} y_{0}+\rho(x) \sum_{i=1}^{K} \int_{0}^{T^{*}-\delta} e^{\Delta\left(T^{*}-\delta-\sigma\right)} \chi_{\omega_{i}} v_{i}(\sigma) d \sigma \\
= & e^{\Delta\left(T^{*}-\delta\right)} y_{0}+\sum_{i=1}^{K} \int_{0}^{T^{*}-\delta} e^{\Delta\left(T^{*}-\delta-\sigma\right)} \rho(x) \chi_{\omega_{i}} u_{i}^{*}(\delta+\sigma) d \sigma \\
& +\int_{0}^{T^{*}-\delta} e^{\Delta\left(T^{*}-\delta-\sigma\right)} \rho(x) \chi_{E_{\delta}^{0}} \chi_{\omega_{i}} w_{\delta}(\sigma) d \sigma \\
= & e^{\Delta\left(T^{*}-\delta\right)} y_{0}+\sum_{i=1}^{K} \int_{\delta}^{T^{*}} e^{\Delta\left(T^{*}-\sigma\right)} \rho(x) \chi_{\omega_{i}} u_{i}^{*}(\sigma) d \sigma+e^{\Delta\left(T^{*}-\delta\right)} h_{\delta} \\
= & e^{\Delta\left(T^{*}-\delta\right)} y_{0}+\sum_{i=1}^{K} \int_{\delta}^{T^{*}} e^{\Delta\left(T^{*}-\sigma\right)} \rho(x) \chi_{\omega_{i}} u_{i}^{*}(\sigma) d \sigma \\
& +\int_{0}^{\delta} e^{\Delta\left(T^{*}-\sigma\right)} \rho(x) \sum_{i=1}^{K} \chi_{\omega_{i}} u_{i}^{*}(\sigma) d \sigma+e^{\Delta T^{*}} y_{0}-e^{\Delta\left(T^{*}-\delta\right)} y_{0} \\
= & e^{\Delta T^{*}} y_{0}+\int_{0}^{T^{*}} e^{\Delta\left(T^{*}-\sigma\right)} \rho(x) \sum_{i=1}^{K} \chi_{\omega_{i}} u_{i}^{*}(\sigma) d \sigma \\
= & 0,
\end{aligned}
$$


which shows that $\left\{v_{i}\right\}$ are the desired controls such that $y\left(\cdot, T^{*}-\delta ;\left\{\chi_{\omega_{i}} \nu_{i}\right\}, y_{0}\right)=0$. It contradicts the definition of $T^{*}$ and we have proved the theorem.

Remark 2.3 There are some relations between the optimal control problem of (1.1) and shape design problem. For more about shape design problem see [8, 14-16].

Before stating the results on Problem (TP2), we define the following reachable set:

$$
\mathcal{R}(T)=\left\{y\left(\cdot, T ; \chi_{\omega} u, y_{0}\right) \mid u \in \mathcal{U}_{\mathrm{ad}}\right\}
$$

for each $T \in(0,+\infty)$.

Theorem 2.4 For any given positive constant $\varepsilon$, Problem (TP2) has a solution $T_{\varepsilon}^{*}$, and $\mathcal{R}\left(T_{\varepsilon}^{*}\right) \cap \bar{B}(0, \varepsilon)$ has only one point belonging to the boundary of $B(0, \varepsilon)$. Moreover, the corresponding time optimal control $u_{\varepsilon}^{*}$ is unique and has the bang-bang property.

Proof Since the proof is long, we separate it into the following several steps.

Step 1. We shall show that there exists at least one time optimal control, i.e., there exists at least one $u^{*} \in \mathcal{U}_{\text {ad }}$ such that $y\left(\cdot, T_{\varepsilon}^{*} ; u^{*}, y_{0}\right) \in \bar{B}(0, \varepsilon)$.

Let $\left\{T_{n}\right\}$ be a monotone decreasing sequence such that $T_{n} \rightarrow T_{\varepsilon}^{*}$ as $n \rightarrow+\infty$, then there exists a sequence $\left\{u_{n}\right\} \subset \mathcal{U}_{\text {ad }}$ such that $y\left(\cdot, T_{n} ; \chi_{\omega} u_{n}, y_{0}\right) \in \bar{B}(0, \varepsilon)$. Set

$$
\tilde{u}_{n}(t)= \begin{cases}u_{n}(t), & t \in\left(0, T_{n}\right), \\ 0, & t \in\left[T_{n}, T_{1}\right) .\end{cases}
$$

Since $M \in L^{\infty}\left(0, T_{1} ; L^{2}(\Omega)\right)$, $\left\{\tilde{u}_{n}\right\}$ is a bounded sequence in $L^{\infty}\left(0, T_{1} ; L^{2}(\Omega)\right)$. Then there exist $\tilde{u}^{*} \in L^{\infty}\left(0, T_{1} ; L^{2}(\Omega)\right)$ and a subsequence of $\left\{u_{n}\right\}$, still so denoted, such that $u_{n} \rightarrow u^{*}$ weakly* in $L^{\infty}\left(0, T_{1} ; L^{2}(\Omega)\right)$. Moreover,

$$
\rho \chi_{\omega} \tilde{u}_{n} \rightarrow \rho \chi_{\omega} \tilde{u}^{*} \quad \text { weakly }{ }^{*} \text { in } L^{\infty}\left(0, T_{1} ; L^{2}(\Omega)\right) \text { as } n \rightarrow \infty .
$$

Therefore the solution $y_{n}\left(\cdot, \cdot ; \chi_{\omega} \tilde{u}_{n}, y_{0}\right)$ to the following system:

$$
\begin{cases}\partial_{t} y_{n}(x, t)-\Delta y_{n}(x, t)=\rho(x) \chi_{\omega}(x) \tilde{u}_{n}(x, t), & \text { in } \Omega \times\left(0, T_{1}\right), \\ y_{n}(x, t)=0, & \text { on } \partial \Omega \times\left(0, T_{1}\right), \\ y_{n}(x, 0)=y_{0}(x), & \text { in } \Omega,\end{cases}
$$

satisfies $y_{n}\left(\cdot, t ; \chi_{\omega} \tilde{u}_{n}, y_{0}\right) \in \bar{B}(0, \varepsilon)$ for $t \geq T_{n}$. Denote by $y^{*}$ the solution to the following system:

$$
\begin{cases}\partial_{t} y^{*}(x, t)-\Delta y^{*}(x, t)=\rho(x) \chi_{\omega}(x) \tilde{u}^{*}(x, t), & \text { in } \Omega \times\left(0, T_{1}\right), \\ y^{*}(x, t)=0, & \text { on } \partial \Omega \times\left(0, T_{1}\right), \\ y^{*}(x, 0)=y_{0}(x), & \text { in } \Omega .\end{cases}
$$


Then

$$
\begin{gathered}
y_{n} \rightarrow y^{*} \quad \text { weakly in } L^{2}\left(0, T ; H_{0}^{1}(\Omega)\right) \cap H^{1}\left(0, T ; L^{2}(\Omega)\right), \\
\text { strongly in } C\left(\left[0, T_{1}\right] ; L^{2}(\Omega)\right) \text { as } n \rightarrow \infty,
\end{gathered}
$$

for any $\delta>0$. Since $y_{n}\left(\cdot, t ; \chi_{\omega} \tilde{u}^{n}, y_{0}\right) \in \bar{B}(0, \varepsilon)$ for $t \geq T_{n}, y^{*}\left(\cdot, t ; \chi_{\omega} \tilde{u}^{*}, y_{0}\right) \in \bar{B}(0, \varepsilon)$ for all $t \geq T_{n}$ and $n \in \mathbb{N}$. Hence $y^{*}\left(\cdot, T_{\varepsilon}^{*} ; \chi_{\omega} \tilde{u}^{*}, y_{0}\right) \in \bar{B}(0, \varepsilon)$. Set

$$
u^{*}(t)=\tilde{u}^{*}(t), \quad t \in\left[0, T_{\varepsilon}^{*}\right]
$$

Then $y^{*}$ satisfies the following system:

$$
\begin{cases}\partial_{t} y^{*}(x, t)-\Delta y^{*}(x, t)=\rho(x) \chi_{\omega}(x) u^{*}(x, t), & \text { in } \Omega \times\left(0, T_{1}\right), \\ y^{*}(x, t)=0, & \text { on } \partial \Omega \times\left(0, T_{1}\right), \\ y^{*}(x, 0)=y_{0}(x), & \text { in } \Omega,\end{cases}
$$

and $y^{*}\left(\cdot, T_{\varepsilon}^{*} ; \chi_{\omega} u^{*}, y_{0}\right) \in \bar{B}(0, \varepsilon)$.

Claim: $\left\|u^{*}(t)\right\|_{L^{2}(\Omega)} \leq M(t)$ for a.e. $t \in\left[0, T_{\varepsilon}^{*}\right]$. Indeed, let $\left\{\zeta_{k}\right\}_{k \in \mathbb{N}}$ be the countable density subset of $L^{2}(\Omega)$. Denote by $\mathcal{L}$ the Lebesgue point of $\left\langle u_{n}(t), \zeta_{k}\right\rangle, t \in\left[0, T_{\varepsilon}^{*}\right]$, where $\langle\cdot, \cdot\rangle$ is the inner product of $u_{n}(t)$ and $\zeta_{k}$ in $L^{2}(\Omega)$. Since $\left\langle u_{n}(t), \zeta_{k}\right\rangle,\left\langle u^{*}(t), \zeta_{k}\right\rangle \in L^{\infty}\left(0, T_{\varepsilon}^{*}\right)$, for each

$$
t_{0} \in E_{0} \equiv \bigcap_{n, k=1}^{\infty} \mathcal{L}\left(\left\langle u_{n}, \zeta_{k}\right\rangle\right) \cap \bigcap_{k=1}^{\infty} \mathcal{L}\left(\left\langle u^{*}, \zeta_{k}\right\rangle\right)
$$

we have

$$
\begin{aligned}
& \lim _{\delta \rightarrow 0} \frac{1}{2 \delta} \int_{t_{0}-\delta}^{t_{0}+\delta}\left\langle u_{n}(t), \zeta_{k}\right\rangle d t=\left\langle u_{n}\left(t_{0}\right), \zeta_{k}\right\rangle, \\
& \lim _{\delta \rightarrow 0} \frac{1}{2 \delta} \int_{t_{0}-\delta}^{t_{0}+\delta}\left\langle u^{*}(t), \zeta_{k}\right\rangle d t=\left\langle u^{*}\left(t_{0}\right), \zeta_{k}\right\rangle .
\end{aligned}
$$

By virtue of

$$
\frac{1}{2 \delta} \int_{t_{0}-\delta}^{t_{0}+\delta}\left\langle u_{n}(t), \zeta_{k}\right\rangle d t \rightarrow \frac{1}{2 \delta} \int_{t_{0}-\delta}^{t_{0}+\delta}\left\langle u^{*}(t), \zeta_{k}\right\rangle d t \quad \text { as } n \rightarrow \infty
$$

$u_{n} \rightarrow u^{*}$ weakly* in $L^{\infty}\left(0, T_{\varepsilon}^{*} ; L^{2}(\Omega)\right)$, and the arbitrary of $\delta>0$, we get

$$
\begin{aligned}
& \left\langle u_{n}\left(t_{0}\right), \zeta_{k}\right\rangle=\lim _{\delta \rightarrow 0} \frac{1}{2 \delta} \int_{t_{0}-\delta}^{t_{0}+\delta}\left\langle u_{n}(t), \zeta_{k}\right\rangle d t \rightarrow \\
& \lim _{\delta \rightarrow 0} \frac{1}{2 \delta} \int_{t_{0}-\delta}^{t_{0}+\delta}\left\langle u^{*}(t), \zeta_{k}\right\rangle d t=\left\langle u^{*}\left(t_{0}\right), \zeta_{k}\right\rangle \quad \text { as } n \rightarrow \infty .
\end{aligned}
$$

Since $\left\{\zeta_{k}\right\}$ is dense in $L^{2}(\Omega)$, we have

$$
\left\langle u_{n}\left(t_{0}\right), \zeta\right\rangle \rightarrow\left\langle u^{*}\left(t_{0}\right), \zeta\right\rangle \quad \text { as } n \rightarrow \infty
$$


for each $\zeta \in L^{2}(\Omega)$. That implies $u_{n}\left(t_{0}\right) \rightarrow u^{*}\left(t_{0}\right)$ weakly in $L^{2}(\Omega)$, and hence $\left\|u^{*}\left(t_{0}\right)\right\|_{L^{2}(\Omega)} \leq$ $\liminf _{n \rightarrow \infty}\left\|u_{n}\left(t_{0}\right)\right\|_{L^{2}(\Omega)} \leq M$. Applying

$$
\left|E_{0}\right|=T_{\varepsilon}^{*}
$$

we obtain $\left\|u^{*}(t)\right\|_{L^{2}(\Omega)} \leq M$ for a.e. $t \in\left[0, T_{\varepsilon}^{*}\right]$. That proves the claim.

Step 2 . We show that $\mathcal{R}\left(T_{\varepsilon}^{*}\right) \cap \bar{B}(0, \varepsilon)$ has only one point. If so, it is obvious that this point belongs to the boundary of $B(0, \varepsilon)$.

In Step 1 we get $\mathcal{R}\left(T_{\varepsilon}^{*}\right) \cap \bar{B}(0, \varepsilon) \neq \emptyset$. By contradiction, we assume that $\mathcal{R}\left(T_{\varepsilon}^{*}\right) \cap \bar{B}(0, \varepsilon)$ has at least two points, i.e., there exist $y_{1} \equiv y\left(\cdot, T_{\varepsilon}^{*} ; \chi_{\omega} u_{1}^{*}, y_{0}\right), y_{2} \equiv y\left(\cdot, T_{\varepsilon}^{*} ; \chi_{\omega} u_{2}^{*}, y_{0}\right) \in \mathcal{R}\left(T_{\varepsilon}^{*}\right) \cap$ $\bar{B}(0, \varepsilon)$ with $y_{1} \neq y_{2}$. It is obvious that $u_{1}^{*} \neq u_{2}^{*}$ in $\mathcal{U}_{\mathrm{ad}}$. Define

$$
\hat{u} \equiv \frac{u_{1}^{*}+u_{2}^{*}}{2}
$$

Since $\hat{u} \in \mathcal{U}_{\mathrm{ad}}$, and $B(0, \varepsilon)$ is strongly convex in $L^{2}(\Omega)$, we get $\hat{y} \equiv y\left(\cdot, T_{\varepsilon}^{*} ; \chi_{\omega} \hat{u}, y_{0}\right)=\frac{1}{2} y_{1}+\frac{1}{2} y_{2}$ is an inner point of $B(0, \varepsilon)$, i.e., there exists $\gamma>0$ such that $B(\hat{y}, \gamma) \subset B(0, \varepsilon)$.

For any $\xi>0$, define

$$
h_{\xi} \equiv \hat{y}-y\left(\cdot, T_{\varepsilon}^{*}-\xi ; \chi_{\omega} \hat{u}, y_{0}\right)
$$

It is easy to check that

$$
\begin{aligned}
h_{\xi}= & e^{\Delta\left(T_{\varepsilon}^{*}-\xi\right)}\left[e^{\Delta \xi}-I\right] y_{0}+\int_{0}^{T_{\varepsilon}^{*}-\xi}\left[e^{\Delta \xi}-I\right] e^{\Delta\left(T_{\varepsilon}^{*}-\xi-\sigma\right)} \rho \chi_{\omega} \hat{u}(\sigma) d \sigma \\
& +\int_{T_{\varepsilon}^{*}-\xi}^{T_{\varepsilon}^{*}} e^{\Delta\left(T_{\varepsilon}^{*}-\sigma\right)} \rho \chi_{\omega} \hat{u}(\sigma) d \sigma .
\end{aligned}
$$

Hence, $\xi$ can be chosen small enough such that $\left\|h_{\xi}\right\|_{L^{2}(\Omega)} \leq \gamma$. Therefore, we can get $y\left(\cdot, T_{\varepsilon}^{*}-\xi ; \chi_{\omega} \hat{u}, y_{0}\right) \in \bar{B}(0, \varepsilon)$. This contradicts the optimal time $T_{\varepsilon}^{*}$. Subsequently, the set $\mathcal{R}\left(T_{\varepsilon}^{*}\right) \cap \bar{B}(0, \varepsilon)$ has only one point, and this point belongs to the boundary of $B(0, \varepsilon)$.

Step 3 . The time optimal control $u^{*}$ has the bang-bang property.

Since $\mathcal{R}\left(T_{\varepsilon}^{*}\right) \cap \bar{B}(0, \varepsilon)$ has only one point (denote this point by $y^{*}=y\left(\cdot, T_{\varepsilon}^{*} ; \chi_{\omega} u^{*}, y_{0}\right)$ ), and $\mathcal{R}\left(T_{\varepsilon}^{*}\right)$ and $\bar{B}(0, \varepsilon)$ are two convex sets, by hyperplane separation theorem, there exists $\eta^{*} \in L^{2}(\Omega)$ such that

$$
\sup _{y \in \mathcal{R}\left(T_{\varepsilon}^{*}\right)}\left\langle y, \eta^{*}\right\rangle \leq \inf _{z \in \bar{B}(0, \varepsilon)}\left\langle z, \eta^{*}\right\rangle \leq\left\langle y^{*}, \eta^{*}\right\rangle
$$

Notice that $y \in \mathcal{R}\left(T_{\varepsilon}^{*}\right)$ can be written by

$$
y\left(\cdot, T_{\varepsilon}^{*} ; \chi_{\omega} u, y_{0}\right)=e^{\Delta T_{\varepsilon}^{*}} y_{0}+\int_{0}^{T_{\varepsilon}^{*}} e^{\Delta\left(T_{\varepsilon}^{*}-\sigma\right)} \rho \chi_{\omega} u(\sigma) d \sigma .
$$

Then (2.11) can be written as

$$
\sup _{\bar{u} \in \mathcal{U}_{1}} \int_{0}^{T_{\varepsilon}^{*}}\left\langle e^{\Delta\left(T_{\varepsilon}^{*}-\sigma\right)} \rho \chi_{\omega} M \bar{u}(\sigma), \eta^{*}\right\rangle d \sigma \leq \int_{0}^{T_{\varepsilon}^{*}}\left\langle e^{\Delta\left(T_{\varepsilon}^{*}-\sigma\right)} \rho \chi_{\omega} M \bar{u}^{*}(\sigma), \eta^{*}\right\rangle d \sigma .
$$


Here

$$
\bar{u}^{*} \in \mathcal{U}_{1} \equiv\left\{\bar{u} \in L^{\infty}\left(0, T_{\varepsilon}^{*} ; L^{2}(\Omega)\right) \mid\|\bar{u}(t)\|_{L^{2}(\Omega)} \leq 1 \text { for a.e. } t \in\left[0, T_{\varepsilon}^{*}\right]\right\},
$$

and

$$
u^{*}(t)=M \bar{u}^{*}(t) \quad \text { for all } t \in\left[0, T_{\varepsilon}^{*}\right] .
$$

Hence, we have

$$
\sup _{\bar{u} \in \mathcal{U}_{1}} \int_{0}^{T_{\varepsilon}^{*}}\left\langle\bar{u}(\sigma), e^{\Delta\left(T_{\varepsilon}^{*}-\sigma\right)} \rho \chi_{\omega} M \eta^{*}\right\rangle d \sigma \leq \int_{0}^{T_{\varepsilon}^{*}}\left\langle\bar{u}^{*}(\sigma), e^{\Delta\left(T_{\varepsilon}^{*}-\sigma\right)} \rho \chi_{\omega} M \eta^{*}\right\rangle d \sigma .
$$

For given $t_{0} \in E_{0}$, choosing

$$
\bar{u}(t)= \begin{cases}\bar{u}^{*}(t), & \text { for } t \in\left(0, T_{\varepsilon}^{*}\right) \backslash\left(t_{0}-\lambda, t_{0}+\lambda\right), \\ \zeta, & \text { for } t \in\left(t_{0}-\lambda, t_{0}+\lambda\right) \subset\left(0, T_{\varepsilon}^{*}\right),\end{cases}
$$

where $\zeta \in L^{2}(\Omega)$, we get

$$
\sup _{\zeta \in L^{2}(\Omega)}\left\langle\zeta, e^{\Delta\left(T_{\varepsilon}^{*}-t_{0}\right)} \rho \chi_{\omega} M \eta^{*}\right\rangle \leq\left\langle\bar{u}^{*}\left(t_{0}\right), e^{\Delta\left(T_{\varepsilon}^{*}-t_{0}\right)} \rho \chi_{\omega} M \eta^{*}\right\rangle,
$$

i.e.,

$$
\begin{aligned}
\left\|e^{\Delta\left(T_{\varepsilon}^{*}-t_{0}\right)} \rho \chi_{\omega} \eta^{*}\right\|_{L^{2}(\Omega)} & =\sup _{\zeta \in L^{2}(\Omega)}\left\langle\zeta, e^{\Delta\left(T_{\varepsilon}^{*}-t_{0}\right)} \rho \chi_{\omega} \eta^{*}\right\rangle \\
& \leq\left\langle\bar{u}^{*}(t), e^{\Delta\left(T_{\varepsilon}^{*}-t_{0}\right)} \rho \chi_{\omega} \eta^{*}\right\rangle \\
& \leq\left\|\bar{u}^{*}(t)\right\|_{L^{2}(\Omega)}\left\|e^{\Delta\left(T_{\varepsilon}^{*}-t_{0}\right)} \rho \chi_{\omega} \eta^{*}\right\|_{L^{2}(\Omega)} .
\end{aligned}
$$

This implies that

$$
\left\|\bar{u}^{*}\left(t_{0}\right)\right\|_{L^{2}(\Omega)}=1
$$

by $\bar{u}^{*} \in \mathcal{U}_{1}$. Equation (2.13), together with (2.12) and $\left|E_{0}\right|=T_{\varepsilon}^{*}$, yields

$$
\left\|u^{*}(t)\right\|_{L^{2}(\Omega)}=M \quad \text { for a.e. } t \in\left[0, T_{\varepsilon}^{*}\right] .
$$

From the above, we get the time optimal control's bang-bang property. That completes the proof.

\section{Norm optimal control problems with multi-time and multi-domain controls}

In this section, let $T \in \mathbb{R}^{+}, K \in \mathbb{Z}^{+}$be given finite constants, and $\Pi^{K}$ be time partition of $[0, T]$ defined by

$$
\Pi_{K}: 0=t_{0} \leq t_{1} \leq t_{2} \leq \cdots \leq t_{K}=T .
$$


For any $i \in\{1,2, \ldots, K\}$, set $I_{i}=\left(t_{i-1}, t_{i}\right]$. Taking $E_{i}=I_{i}$, we can rewrite $(1.1)$ as

$$
\begin{cases}\partial_{t} y(x, t)-\Delta y(x, t)=\rho(x) \sum_{i=1}^{K} \chi_{I_{i}}(t) \chi_{\omega_{i}}(x) u_{i}(x, t), & \text { in } \Omega \times(0, T), \\ y(x, t)=0, & \text { on } \partial \Omega \times(0, T), \\ y(x, 0)=y_{0}(x), & \text { in } \Omega .\end{cases}
$$

It is obvious that the system is null controllable (see $[5,17])$. For any given partition $\Pi^{K}$, by standard minimizing sequence method, there exists a solution to the following norm optimal control problem:

$$
\begin{aligned}
N\left(\Pi_{K}\right) \equiv & \inf \left\{\sum_{i=1}^{K}\left\|\rho \chi_{I_{i}} \chi_{\omega_{i}} u_{i}\right\|_{L^{\infty}\left(0, T ; L^{2}(\Omega)\right)} \mid y\left(\cdot, T ;\left\{\chi_{I_{i}} \chi_{\omega_{i}} u_{i}\right\}, y_{0}\right)=0,\right. \\
& \left.\left\{u_{i}\right\}_{i=1}^{K} \subset L^{\infty}\left(0, T ; L^{2}(\Omega)\right)\right\} .
\end{aligned}
$$

We are interested in the partition's existence of the following norm optimal control problem:

\section{Problem (NP)}

$$
N_{K}^{*} \equiv \inf \left\{N\left(\Pi_{K}\right) \mid \Pi_{K} \text { is defined by partition (3.1) }\right\}
$$

We have the following solvability result on Problem (NP).

Theorem 3.1 For any $K>1$, there exists at least one solution to Problem (NP).

Proof It is obviously that $N_{K}^{*}<\infty$. Let $\left\{\Pi_{K}^{n}\right\}$ be the partition sequence such that

$$
N\left(\Pi_{K}^{n}\right) \rightarrow N_{K}^{*} \quad \text { as } n \rightarrow \infty .
$$

Then there exists a control sequence $\left\{u_{1}^{n}, u_{2}^{n}, \ldots, u_{K}^{n}\right\}_{n=1}^{\infty}$, such that

$$
\begin{cases}\partial_{t} y^{n}(x, t)-\Delta y^{n}(x, t)=\rho(x) \sum_{i=1}^{K} \chi_{I_{i}^{n}}(t) \chi_{\omega_{i}}(x) u_{i}^{n}(x, t), & \text { in } \Omega \times(0, T) \\ y^{n}(x, t)=0, & \text { on } \partial \Omega \times(0, T), \\ y^{n}(x, 0)=y_{0}(x), y^{n}(x, T)=0, & \text { in } \Omega,\end{cases}
$$

with

$$
\left\|\rho \sum_{i=1}^{K} \chi_{I_{i}^{n}} \chi_{\omega_{i}} u_{i}^{n}\right\|_{L^{\infty}\left(0, T ; L^{2}(\Omega)\right)}=N\left(\Pi_{K}^{n}\right) \quad \text { for all } n \in \mathbb{N}
$$

Since $\left\{t_{1}^{n}\right\} \subset[0, T]$ is a bounded sequence, there exist a subsequence of $\left\{t_{1}^{n}\right\}$, still so denoted, and $t_{1}^{0}$ such that $t_{1}^{n} \rightarrow t_{1}^{0}$ as $n \rightarrow \infty$. Also, there exist a subsequence of $\left\{t_{2}^{n}\right\}$, still so denoted, and $t_{2}^{0}$ satisfying $t_{2}^{n} \rightarrow t_{2}^{0}$ as $n \rightarrow \infty$. Moreover, since $t_{1}^{n} \leq t_{2}^{n}$ for all $n \in \mathbb{N}$, we have $t_{1}^{0} \leq t_{2}^{0}$. By the same line, we can get $\left\{t_{i}^{0}\right\}_{i=1}^{K}$ satisfying

$$
0=t_{0}^{0} \leq t_{1}^{0} \leq t_{2}^{0} \leq \cdots \leq t_{K}^{0}=T
$$


Denote the above partition by $\Pi_{K}^{0}$. We also can define $I_{i}^{0}$ for each $i \in\{1,2, \ldots, K\}$. Naturally, one has

$$
\left|I_{i}^{n} \triangle I_{i}^{0}\right| \rightarrow 0 \quad \text { as } n \rightarrow \infty
$$

Here and in what follows, we define $I_{i}^{n} \triangle I_{i}^{0} \equiv\left(I_{i}^{n}-I_{i}^{0}\right) \cup\left(I_{i}^{0}-I_{i}^{n}\right)$ for all $i, n$.

On the other hand, since $\left\{u_{1}^{n}, u_{2}^{n}, \ldots, u_{K}^{n}\right\}_{n=1}^{\infty}$ is bounded, $\left\{u_{i}^{n}\right\}_{n=1}^{\infty}$ is also a bounded sequence in $L^{\infty}\left(0, T ; L^{2}(\Omega)\right)$ for all $i=1, \ldots, K$. Then, for each $i=1, \ldots, K$, there exist a subsequence of $\left\{u_{i}^{n}\right\}_{n=1}^{\infty}$, still so denoted, and $u_{i}^{0} \in L^{\infty}\left(0, T ; L^{2}(\Omega)\right)$ such that

$$
u_{i}^{n} \rightarrow u_{i}^{0} \quad \text { weakly* in } L^{\infty}\left(0, T ; L^{2}(\Omega)\right) \text { as } n \rightarrow \infty .
$$

For each $v \in L^{1}\left(0, T ; L^{2}(\Omega)\right)$, we have

$$
\begin{aligned}
\int_{0}^{T} & \int_{\Omega} \rho\left(\chi_{I_{i}^{n}} \chi_{\omega_{i}} u_{i}^{n}-\chi_{I_{i}^{0}} \chi_{\omega_{i}} u_{i}^{0}\right) v d x d t \\
= & \int_{0}^{T} \int_{\Omega} \rho\left(\chi_{I_{i}^{n}} \chi_{\omega_{i}} u_{i}^{n}-\chi_{I_{i}^{0}} \chi_{\omega_{i}} u_{i}^{n}\right) v d x d t \\
& +\int_{0}^{T} \int_{\Omega} \rho\left(\chi_{I_{i}^{0}} \chi_{\omega_{i}} u_{i}^{n}-\chi_{I_{i}^{0}} \chi_{\omega_{i}} u_{i}^{0}\right) v d x d t \\
= & \int_{0}^{T} \int_{\Omega} \rho\left(\chi_{I_{i}^{n}}-\chi_{I_{i}^{0}}\right) \chi_{\omega_{i}} u_{i}^{n} v d x d t \\
& +\int_{0}^{T} \int_{\Omega} \rho\left(u_{i}^{n}-u_{i}^{0}\right) \chi_{I_{i}^{0}} \chi_{\omega_{i}} v d x d t .
\end{aligned}
$$

By (3.6), we get

$$
\int_{0}^{T} \int_{\Omega}\left(u_{i}^{n}-u_{i}^{0}\right) \rho \chi_{I_{i}^{0}} \chi_{\omega_{i}} v d x d t \rightarrow 0 \quad \text { as } n \rightarrow \infty .
$$

By (3.5) and the absolutely continuity of $v \in L^{1}\left(0, T ; L^{2}(\Omega)\right)$, we have

$$
\begin{aligned}
& \left|\int_{0}^{T} \int_{\Omega} \rho\left(\chi_{I_{i}^{n}}-\chi_{I_{i}^{0}}\right) \chi_{\omega_{i}} u_{i}^{n} v d x d t\right| \\
& \quad \leq\left\|u_{i}^{n}\right\|_{L^{\infty}\left(0, T ; L^{2}(\Omega)\right)}\left\|\rho\left(\chi_{I_{i}^{n}}-\chi_{I_{i}^{0}}\right) \chi_{\omega_{i}} v\right\|_{L^{1}\left(0, T ; L^{2}(\Omega)\right)} \\
& \quad=\left\|u_{i}^{n}\right\|_{L^{\infty}\left(0, T ; L^{2}(\Omega)\right)} \int_{I_{i}^{n} \Delta I_{i}^{0}}\left(\int_{\Omega}\left|\rho \chi_{\omega_{i}} v\right|^{2} d x\right)^{\frac{1}{2}} d t \\
& \quad \leq\left\|u_{i}^{n}\right\|_{L^{\infty}\left(0, T ; L^{2}(\Omega)\right)} \int_{I_{i}^{n} \Delta I_{i}^{0}}\left(\int_{\Omega}|v|^{2} d x\right)^{\frac{1}{2}} d t \\
& \quad \rightarrow 0 \quad \text { as } n \rightarrow \infty .
\end{aligned}
$$

Equation (3.7), together with (3.8) and (3.9), yields

$$
\int_{0}^{T} \int_{\Omega} \rho\left(\chi_{I_{i}^{n}} \chi_{\omega_{i}} u_{i}^{n}-\chi_{I_{i}^{0}} \chi_{\omega_{i}} u_{i}^{0}\right) v d x d t \rightarrow 0 \quad \text { as } n \rightarrow \infty .
$$


That implies, for all $i \in\{1, \ldots, K\}$,

$$
\rho \chi_{I_{i}^{n}} \chi_{\omega_{i}} u_{i}^{n} \rightarrow \rho \chi_{I_{i}^{0}} \chi_{\omega_{i}} u_{i}^{0} \quad \text { weakly* in } L^{\infty}\left(0, T ; L^{2}(\Omega)\right) \text { as } n \rightarrow \infty \text {. }
$$

Therefore, one gets

$$
\rho \sum_{i=1}^{K} \chi_{I_{i}^{n}} \chi_{\omega_{i}} u_{i}^{n} \rightarrow \rho \sum_{i=1}^{K} \chi_{I_{i}^{0}} \chi_{\omega_{i}} u_{i}^{0} \quad \text { weakly* in } L^{\infty}\left(0, T ; L^{2}(\Omega)\right) \text { as } n \rightarrow \infty
$$

and

$$
\begin{aligned}
& \left\|\rho \sum_{i=1}^{K} \chi_{I_{i}^{0}} \chi_{\omega_{i}} u_{i}^{0}\right\|_{L^{\infty}\left(0, T ; L^{2}(\Omega)\right)} \\
& \quad \leq \liminf _{n \rightarrow \infty}\left\|\rho \sum_{i=1}^{K} \chi_{I_{i}^{n}} \chi_{\omega_{i}} u_{i}^{n}\right\|_{L^{\infty}\left(0, T ; L^{2}(\Omega)\right)} \\
& \quad=N_{K}^{*} .
\end{aligned}
$$

Let $y^{0}$ be the solution to the following system:

$$
\begin{cases}\partial_{t} y^{0}(x, t)-\Delta y^{0}(x, t)=\rho(x) \sum_{i=1}^{K} \chi_{I_{i}^{0}}(t) \chi_{\omega_{i}}(x) u_{i}^{0}(x, t), & \text { in } \Omega \times(0, T), \\ y^{0}(x, t)=0, & \text { on } \partial \Omega \times(0, T), \\ y^{0}(x, 0)=y_{0}(x), & \text { in } \Omega .\end{cases}
$$

By (3.10) we get

$$
\begin{aligned}
y^{n} \rightarrow y^{0} & \text { weakly in } L^{2}\left(0, T ; H_{0}^{1}(\Omega)\right) \cap H^{1}\left(0, T ; L^{2}(\Omega)\right), \\
& \text { strongly in } C\left([\delta, T] ; L^{2}(\Omega)\right),
\end{aligned}
$$

for every $\delta>0$. Since $y^{n}(T)=0$, immediately we have

$$
y^{0}(T)=0
$$

Combining (3.12), (3.13) and (3.11), we complete the proof.

Now, let us consider an alteration of system (3.2):

$$
\begin{cases}\partial_{t} y(x, t)-\Delta y(x, t)=\rho(x) \sum_{i=1}^{K} \chi_{I_{i}}(t) \chi_{\omega_{\sigma(i)}}(x) u_{i}(x, t), & \text { in } \Omega \times(0, T), \\ y(x, t)=0, & \text { on } \partial \Omega \times(0, T), \\ y(x, 0)=y_{0}(x), & \text { in } \Omega,\end{cases}
$$

where

$$
\sigma:\{1, \ldots, K\} \rightarrow\{1, \ldots, K\} \quad \text { is a map. }
$$


By the aforementioned discussion, this system is null controllable, and for given partition $\Pi_{K}$ and map $\sigma$, there exists at least a solution to the following norm optimal control problem:

$$
\begin{aligned}
N(\sigma) \equiv & \inf \left\{\sum_{i=1}^{K}\left\|\rho \chi_{I_{i}} \chi_{\omega_{\sigma(i)}} u_{i}\right\|_{L^{\infty}\left(0, T ; L^{2}(\Omega)\right)} \mid y\left(\cdot, T ;\left\{\chi_{I_{i}} \chi_{\omega_{\sigma(i)}} u_{i}\right\}, y_{0}\right)=0,\right. \\
& \left.\left\{u_{i}\right\}_{i=1}^{K} \subset L^{\infty}\left(0, T ; L^{2}(\Omega)\right)\right\} .
\end{aligned}
$$

Let us consider the solvability of the following norm optimal control problem:

$$
N \equiv \inf \{N(\sigma) \mid \sigma \text { is defined by (3.14) }\} .
$$

Since the number of $\sigma$ is finite, it is obvious that there exists $\sigma^{*}$ satisfying $N^{*}=N\left(\sigma^{*}\right)$. Hence, we obtain the following result.

Proposition 3.2 Let $\left\{I_{i}\right\}_{i=1}^{K},\left\{\omega_{k}\right\}_{k=1}^{K}$ be defined as before. Then there exists at least a solution to the problem (3.15).

\section{Acknowledgements}

The authors are grateful to the anonymous referees for helpful comments and suggestions, which greatly improved the presentation of this paper.

\section{Competing interests}

The authors declare that there are no competing interests regarding the publication of this article.

\section{Authors' contributions}

All authors read and approved the final manuscript.

\section{Publisher's Note}

Springer Nature remains neutral with regard to jurisdictional claims in published maps and institutional affiliations.

Received: 28 April 2017 Accepted: 22 September 2017 Published online: 11 October 2017

References

1. Fattorini, HO: Infinite-Dimensional Optimization and Control Theory. Encyclopedia of Mathematics and Its Applications, vol. 62. Cambridge University Press, Cambridge (1999)

2. Lü, Q, Zhang, X: General Pontryagin-Type Stochastic Maximum Principle and Backward Stochastic Evolution Equations in Infinite Dimensions. SpringerBriefs in Mathematics. Springer, Cham (2014)

3. Yong, J, Zhou, XY: Stochastic Controls: Hamiltonian Systems and HJB Equations. Applications of Mathematics (New York), vol. 43. Springer, New York (1999)

4. Fattorini, HO: Time and norm optimal controls: a survey of recent results and open problems. Acta Math. Sci. Ser. B Engl. Ed. 31, 2203-2218 (2011)

5. Apraiz, J, Escauriaza, L, Wang, G, Zhang, C: Observability inequalities and measurable sets. J. Eur. Math. Soc. 16, 2433-2475 (2014)

6. Guo, B-Z, Xu, Y, Yang, D-H: Optimal actuator location of minimum norm controls for heat equation with general controlled domain. J. Differ. Equ. 261, 3588-3614 (2016)

7. Guo, B-Z, Yang, D-H, Zhang, L: On optimal location of diffusion and related optimal control for null controllable heat equation. J. Math. Anal. Appl. 433, 1333-1349 (2016)

8. Guo, B-Z, Yang, D-H: Optimal actuator location for time and norm optimal control of null controllable heat equation. Math. Control Signals Syst. 27, 23-48 (2015)

9. Wang, G: $L^{\infty}$-null controllability for the heat equation and its consequences for the time optimal control problem. SIAM J. Control Optim. 47, 1701-1720 (2008)

10. Wang, $G, X u, Y, Z$ hang, $Y$ : Attainable subspaces and the bang-bang property of time optimal controls for heat equations. SIAM J. Control Optim. 53, 592-621 (2015)

11. Wang, Y, Yang, D-H, Yong, J, Yu, Z: Exact controllability of linear stochastic differential equations and related problems. Math. Control Relat. Fields 7(2), 305-345 (2017)

12. Wang, Y, Zhang, C: The norm optimal control problem for stochastic linear control systems. ESAIM Control Optim. Calc. Var. 21, 399-413 (2015) 
13. Yang, D-H, Zhong, J: Observability inequality of backward stochastic heat equations for measurable sets and its applications. SIAM J. Control Optim. 54, 1157-1175 (2016)

14. Guo, B-Z, Yang, D-H: On convergence of boundary Hausdorff measure and application to a boundary shape optimization problem. SIAM J. Control Optim. 51, 253-273 (2013)

15. Guo, B-Z, Yang, D-H: Some compact classes of open sets under Hausdorff distance and application to shape optimization. SIAM J. Control Optim. 50, 222-242 (2012)

16. Yang, D-H: Shape optimization of stationary Navier-Stokes equation overclasses of convex domains. Nonlinear Anal. 71, 6202-6211 (2009)

17. Zuazua, E: Controllability of partial differential equations. Manuscript (2006)

Submit your manuscript to a SpringerOpen ${ }^{\circ}$ journal and benefit from:

- Convenient online submission

Rigorous peer review

- Open access: articles freely available online

- High visibility within the field

- Retaining the copyright to your article

Submit your next manuscript at $\mathbf{s p r i n g e r o p e n . c o m ~}$ 